\title{
Altered Expression of GABAergic Markers in the Forebrain of Young and Adult Engrailed-2 Knockout Mice
}

\author{
Giovanni Provenzano ${ }^{1, *}$, Angela Gilardoni ${ }^{1}$, Marika Maggia ${ }^{1}$, Mattia Pernigo ${ }^{1}$, Paola Sgadò ${ }^{2}$, \\ Simona Casarosa ${ }^{1,3}[$ and Yuri Bozzi $2,3, *[1$ \\ 1 Department of Cellular, Computational and Integrative Biology (CIBIO), University of Trento, \\ 38068 Rovereto, Italy; angela.gilardoni@yahoo.it (A.G.); marika.maggia@gmail.com (M.M.); \\ perni.buz@gmail.com (M.P.); simona.casarosa@unitn.it (S.C.) \\ 2 Center for Mind/Brain Sciences (CIMeC), University of Trento, 38068 Rovereto, Italy; paola.sgado@unitn.it \\ 3 C.N.R. Neuroscience Institute, 5624 Pisa, Italy \\ * Correspondence: giovanni.provenzano@unitn.it (G.P.); yuri.bozzi@unitn.it (Y.B.); \\ Tel.: +39-0461-283589 (G.P.); +39-0464-808707 (Y.B.)
}

Received: 23 February 2020; Accepted: 30 March 2020; Published: 1 April 2020

\begin{abstract}
Impaired function of GABAergic interneurons, and the subsequent alteration of excitation/inhibition balance, is thought to contribute to autism spectrum disorders (ASD). Altered numbers of GABAergic interneurons and reduced expression of GABA receptors has been detected in the brain of ASD subjects and mouse models of ASD. We previously showed a reduced expression of GABAergic interneuron markers parvalbumin (PV) and somatostatin (SST) in the forebrain of adult mice lacking the Engrailed2 gene (En2 ${ }^{--}$mice). Here, we extended this analysis to postnatal day (P) 30 by using in situ hybridization, immunohistochemistry, and quantitative RT-PCR to study the expression of GABAergic interneuron markers in the hippocampus and somatosensory cortex of En2 ${ }^{-/}$ and wild type (WT) mice. In addition, GABA receptor subunit mRNA expression was investigated by quantitative RT-PCR in the same brain regions of P30 and adult En2 ${ }^{--}$and WT mice. As observed in adult animals, PV and SST expression was decreased in En $2^{-/}$forebrain of P30 mice. The expression of GABA receptor subunits (including the ASD-relevant Gabrb3) was also altered in young and adult $E n 2^{-/}$forebrain. Our results suggest that GABAergic neurotransmission deficits are already evident at P30, confirming that neurodevelopmental defects of GABAergic interneurons occur in the En2 mouse model of ASD.
\end{abstract}

Keywords: interneuron; parvalbumin; somatostatin; GABA; receptor; hippocampus; somatosensory; autism; mouse; development

\section{Introduction}

Impaired function of GABAergic interneurons and subsequent alteration of excitation/inhibition balance in neural circuits has been implicated in neurodevelopmental disorders [1]. Forebrain GABAergic interneurons originate from the ganglionic eminences of the ventral forebrain, from where they migrate and differentiate during embryonic and early postnatal development [2]. Different classes of GABAergic interneurons are distinguished on the basis of their morphology, neurochemical content, intrinsic electrophysiological properties, and connectivity [3-7]. Defects in GABAergic neuron number and transmission have been hypothesized to contribute to autism spectrum disorders (ASD) [8-10]. Altered numbers of GABAergic interneurons were detected in the hippocampus of ASD subjects [11], and reduced GABA receptor density, number, and protein expression were also observed in the cerebellum and cortical areas of people with ASD [12]. Interneuron defects have also 
been reported in mouse models of ASD [10], suggesting that defects in the development and function of specific subsets of GABAergic interneurons might be responsible for behavioral impairment in ASD.

Genome-wide association studies identified En2 as a candidate gene for ASD [13]; accordingly, mice lacking En2 [14] display cerebellar defects resembling those reported in ASD subjects [15]. In addition, $E n 2^{--}$mice present a series of pathological behaviors that are reminiscent of some features of ASD individuals, including reduced social interactions, defective spatial learning, and sensory processing deficits [16-19]. As frequently observed in ASD patients, En2 ${ }^{-/}$mice display an increased susceptibility to seizures $[10,20]$ accompanied by the altered expression of ASD- and seizure-relevant genes [21]. We attributed this hyperexcitability to a reduced inhibitory innervation in forebrain areas of $E n 2^{--}$mice, and were able to demonstrate that adult $E n 2^{--}$mice do show a reduced number of parvalbumin (PV)-, somatostatin (SST)-, and neuropeptide Y (NPY)-positive interneurons in the hippocampus, somatosensory, and visual cortex [18,22,23].

Here, we investigated the expression of GABAergic markers parvalbumin (PV) and somatostatin (SST) and GABA receptor subunits in the hippocampus and somatosensory cortex of young En2 ${ }^{-/}$mice. Our results suggest that GABAergic neurotransmission deficits are already evident at an early postnatal age, confirming that neurodevelopmental defects of GABAergic interneurons occur in the En2 mouse model of ASD.

\section{Materials and Methods}

\subsection{Animals}

Experiments were approved by the Animal Welfare Committee of the University of Trento and Italian Ministry of Health (projects 949/2015-PR and 847/2018-PR), in accordance to the European Community Directive 2010/63/EU. Animals were housed in a $12 \mathrm{hr}$ light/dark cycle with food and water available ad libitum, and all efforts were made to minimize suffering during experimental procedures. The generation and genetic background of En2 mutants were previously described [14,22]. Wild type (WT) and $E n 2^{-}$littermates used in this study were generated via heterozygous mating and genotyped as described [22]. A total of 44 age-matched young littermates (22 mice per genotype; 30 days old; weight $=20-25 \mathrm{~g}$ ) of both sexes were used. Eight mice (4 per genotype) were used for histological morphometric analyses, 8 mice (4 per genotype) for in situ hybridization, 10 mice ( 5 per genotype) for immunohistochemistry, and 18 mice (9 per genotype) for quantitative RT-PCR experiments. An additional group of 8 age-matched adult littermates (4 mice per genotype; 3-5 months old; weight $=25-35 \mathrm{~g}$ ) of both sexes were used for quantitative RT-PCR. Previous studies showed that similar group sizes are sufficient to obtain statistically significant results in histological, immunohistochemical, in situ hybridization, and RT-PCR studies [20,22]. All experiments were performed blind to genotype. Mice were assigned a numerical code by an operator who did not take part in the experiments, and codes were associated to genotypes only at the moment of data analysis. Experiments were performed on mice of both sexes, since previous studies did not reveal major significant differences between sexes at molecular, anatomical, and behavioral level [17,19,22].

\subsection{Morphometric Analysis}

Bright-field images of the hippocampus and somatosensory cortex were acquired at 10x primary magnification using a Zeiss M2 AxioImager microscope and merged using Adobe Photoshop software. Morphometric analysis of hippocampal and cortical layers was performed as described [22] on 2 to 4 cresyl-violet (Nissl) stained sections per animal, taken at the level of the dorsal hippocampus ( $n=4$ per genotype). Brain areas were identified according to the Allen Mouse Brain Atlas.

\subsection{In Situ Hybridization}

Brains from $4 \mathrm{WT}$ and $4 \mathrm{En2^{-- }}$ P30 mice were rapidly removed and frozen on dry ice. Twenty micrometer thick coronal sections were cut at the cryostat and fixed in $4 \%$ 
paraformaldehyde. Non-radioactive in situ hybridization was performed as described [20,22] using a mix containing glutamic acid decarboxylase (GAD) 65 (GenBank ID: M72422) and 67 (Genbank ID: NM_017007) digoxigenin-labeled riboprobes. Signal was detected by alkaline phosphatase-conjugated anti-digoxigenin antibody followed by NBT (p-nitroblue tetrazolium chloride)/BCIP (5-bromo-4-chloro-3-indolyl phosphate) alkaline phosphatase staining.

\subsection{Quantitative RT-PCR}

Total RNAs were extracted from dissected hippocampi and somatosensory cortices from 18 young (30 days old; 9 per genotype) and 8 adult (3-5 months old; 4 per genotype) mice, and retrotranscribed to cDNA according to published protocols [22,24]. qRT-PCR was performed in a C1000 Thermal Cycler (Bio-Rad), using the Kapa Probe Fast qPCR Master Mix (Resnova). The mouse housekeeping RNA for mitochondrial ribosomal protein L41 (mRPL41) was used as a standard for quantification. Primers (Eurofins Genomics) were designed on different exons to avoid amplification of genomic DNA (Table 1). Expression analyses were performed using the CFX3 Manager 3.0 (Bio-Rad) software [22,24]. Briefly, mean cycle threshold $(\mathrm{Ct})$ values from triplicate experiments were calculated for each marker and L41, corrected for PCR efficiency and inter-run calibration. The expression level of each marker was then normalized to that of L41 (marker/L41 ratios) for WT and $E n 2^{--}$mice.

Table 1. Primers used for quantitative RT-PCR experiments.

\begin{tabular}{cccc}
\hline Gene Name & Genbank \# & Forward Primer $\mathbf{( 5}^{\prime} \mathbf{- 3}^{\prime} \mathbf{)}$ & Reverse Primer $\mathbf{( 5}^{\prime} \mathbf{-} \mathbf{3}^{\prime} \mathbf{)}$ \\
\hline$L 41$ & NM_001031808.2 & GGTTCTCCCTTTCTCCCTTG & GCACCCCGACTCTTAGTGAA \\
$P V$ & NM_013645 & TGCTCATCCAAGTTGCAGG & GCCACTTTTGTCTTTGTCCAG \\
SOM & NM_009215 & AGGACGAGATGAGGCTGG & CAGGAGTTAAGGAAGAGATATGGG \\
Gabra1 & NM_010250 & CTCTCCCACACTTTTCTCCC & CCGACAGTGTGCTCAGAATG \\
Gabra2 & NM_008066.4 & AGATTCAAAGCCACTGGAGG & CCAGCACCAACCTGACTG \\
Gabra3 & NM_001357816.1 & CAGACTGAGATAGGGACTAGGAG & AGACAGCAACTTGAAGAGACC \\
Gabra5 & NM_176942.4 & CCCTATCCCAACACCTGAAC & AATGTTCAAAGGGTTCTGCC \\
Gabrg2 & NM_008073.4 & CACCGGGCATGAATGTG & GGATGGTACACGCAGAGATG \\
Gabrb2 & NM_001347314 & TCAGAGGATGACTTTGCTA & GCACACAATAATGTTTACTAT \\
Gabrb3 & NM_008071.3 & GAGGTCTTCACAAGCTCAAAATC & AGGCAGGGTAATATTTCACTCAG \\
Gabbr2 & NM_031802.1 & ACATGCAAAGACCCCATAGAG & TCGTGAGAGTAAGACCGTCG \\
\hline
\end{tabular}

\subsection{Immunohistochemistry}

Brains were fixed by transcardial perfusion (4\% paraformaldehyde) followed by post-fixation ( $1 \mathrm{~h}$ at $4{ }^{\circ} \mathrm{C}$ ), and vibratome sections (50 $\mu \mathrm{m}$ thickness) were prepared. Serial sections at the level of the dorsal hippocampus were incubated overnight with the following antibodies; anti-parvalbumin (PV) mouse monoclonal (P3088, Sigma-Aldrich, USA; 1:2000 dilution); anti-somatostatin (SOM) rabbit polyclonal (T-4102, Bachem, UK; 1:2000 dilution); anti-GABA $\mathrm{A}_{\mathrm{A}}$ receptor $\alpha 1$ subunit (GABRA1) rabbit polyclonal

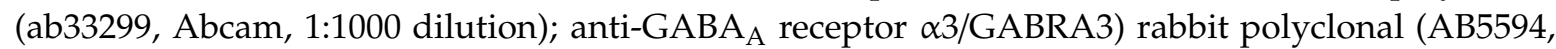
Millipore, 1:1000 dilution); and anti-GABA ${ }_{A}$ receptor $\alpha 5$ (GABRA5) rabbit polyclonal (ab 10098, Abcam, 1:1000 dilution). Biotin-conjugated secondary antibody and streptavidin conjugated to appropriate fluorophores (AlexaFluor 488/594, Invitrogen Life Technologies, USA) were used to reveal the signal.

\subsection{Cell Counts}

Cell counts were performed on acquired images of the hilus and somatosensory cortex, using the ImageJ software (imagej.nih.gov/ij/index.html), as described [22]. Brain areas were identified according to the Allen Mouse Brain Atlas (http://mouse.brain-map.org). For in situ hybridization, counts of GAD65/67 mRNA labeled cells were performed on 3 sections per animal ( $n=4$ per genotype) at the level of the dorsal hippocampus/somatosensory cortex. For each section, bright-field images were acquired at 10× primary magnification using a Zeiss M2 AxioImager microscope and merged using the Zeiss software. For immunohistochemistry, 3 to 4 immunolabeled sections were analyzed per animal ( 5 mice per genotype). For each section, images were acquired at $20 \times$ primary magnification using a Zeiss Axio Observer z1, using the MosaiX and Z-Stack modules of the Zeiss AxioVision software 
(v4.3.1). The same counting procedure was followed for both in situ hybridization (GAD65/67) and immunohistochemistry (PV/SST) images. To count positive cells in the hilus, the total hilar area was measured excluding the granule cell layer, and did not differ between WT and $\mathrm{En}^{-/-}$mice. Cell densities were plotted as the number of positive cells $/ 0.1 \mathrm{~mm}^{2}$. For the somatosensory cortex, cell densities were separately counted in superficial (II-III) and deep (V-VI) layers using at least three counting frames $(200 \times 600 \mu \mathrm{m})$ per section, and plotted as the number of positive cells/counting area. All counts were performed by two independent experimenters blind of genotypes.

\subsection{Statistical Analyses}

Statistical analyses were performed by Prism 6 (GraphPad) software. Student's t-test or two-way ANOVA followed by appropriate post hoc test (as indicated) were used, with statistical significance level set at $p<0.05$.

\section{Results}

Previous studies from our laboratory showed the reduced expression of GABAergic markers in the hippocampus and somatosensory cortex of adult (3-5 months old) En2 ${ }^{-/}$mice, as compared with age-matched controls [20,22]. Here, we extended this analysis to an earlier stage of postnatal development (postnatal day 30, P30).

\subsection{Normal Layering of Hippocampus and Somatosensory Cortex in Young En2 Mutant Mice}

We first performed a morphometric analysis on dorsal hippocampal and somatosensory cortex sections stained with cresyl violet to investigate the layering of these structures in P30 WT and $E n 2^{--}$mice. A normal anatomical layer structure was detected in both WT and En2 ${ }^{--}$mice in the two brain areas analyzed. Total hippocampal thickness did not differ between the two genotypes (WT: $1091 \pm 21 \mu \mathrm{m}, n=17$ sections from 4 mice; En2 $2^{--}: 1090 \pm 18 \mu \mathrm{m}, n=11$ sections from 4 mice). Similarly, the total thickness of the somatosensory cortex (WT, $1253.54 \pm 27 \mu \mathrm{m}, n=15$ sections from 4 mice; En2 $2^{-/}$, $1260.10 \pm 34 \mu \mathrm{m}, n=19$ sections from 4 mice $)$ did not differ between WT and En $2^{--}$mice $(p>0.05$, Student's $t$-test). No difference was detected in hippocampal (Figure 1A) and cortical (Figure 1B) layer thickness between the two genotypes ( $p>0.05$, two-way ANOVA followed by Holm-Sidak test).

We next investigated the expression of GABAergic interneuron markers in the hippocampus of P30 WT and En2 ${ }^{--}$mice. GABAergic interneurons are characterized by the expression of specific markers [3-7]. The total number of GABAergic neurons, estimated by in situ hybridization for GAD65/67 mRNA, was unchanged in the hilus and somatosensory cortex (Figure 2A,B) of En2 ${ }^{-/}$mice, as compared to WT controls.

We previously reported a significantly reduced expression of parvalbumin (PV) and somatostatin (SST) GABAergic interneuron markers in both hippocampus and somatosensory cortex of adult $E n 2^{--}$mice, as compared with age-matched WT animals [22]. Immunohistochemistry experiments performed on brain sections from P30 mice revealed a comparable number of PV-positive cells in both hilus and somatosensory cortex (layers II-III and V-VI) of P30 En2 ${ }^{-/}$mice (Figure 3A,C; $p>0.05$, two-way ANOVA followed by Holm-Sidak test); similarly, the number of hilar SST-positive interneurons did not differ between the two genotypes (Figure 3B,E; $p>0.05$, two-way ANOVA followed by Holm-Sidak test). A statistically significant reduction of SST interneurons was instead detected in layers II-III and V-VI of the En $2^{--}$somatosensory cortex $(-25 \%)$, as compared to age-matched WT controls (Figure $3 \mathrm{~B}, \mathrm{E} ;{ }^{* * *} p<$ 0.001, two-way ANOVA followed by Holm-Sidak test). Quantitative RT-PCR on total RNAs extracted from WT and $E n 2^{-/-}$hippocampi and somatosensory cortices showed contrasting data with respect to immunohistochemistry experiments. PV mRNA levels were significantly reduced in the En2 ${ }^{-/}$ hippocampus $(-55 \%)$ and somatosensory cortex $(-27 \%)$ as compared with WT controls (Figure 3D; ${ }^{*} p<0.05,{ }^{* * *} p<0.001$, two-way ANOVA followed by Holm-Sidak test). Conversely, SST mRNA levels did not differ between the two genotypes in both brain areas analyzed (Figure 3F; $p>0.05$, two-way ANOVA followed by Holm-Sidak test). 


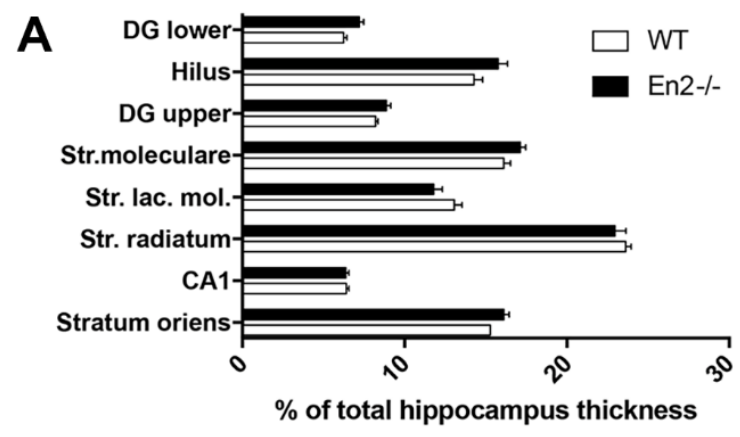

B

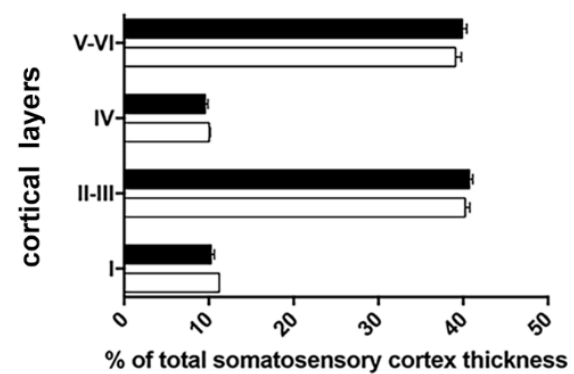

Figure 1. Altered expression of GABAergic interneuron markers in young En2 mutant mice. Normal layering of the young En2 ${ }^{--}$hippocampus and somatosensory cortex. Morphometric analysis of hippocampal (A) and somatosensory cortex (B) layers in young (P30) WT (wild type) and En2 ${ }^{--}$mice ( $n=4$ mice per genotype). Layer thickness is plotted as $\%$ of total thickness of the dorsal hippocampus (A) and somatosensory cortex (B). Genotypes and layers are as indicated; cortical layers are indicated in roman numbers. Abbreviations: DG lower: lower blade of the dentate gyrus; DG upper: upper blade of the dentate gyrus; Str.: stratum; Str. lac. mol.: stratum lacunosum moleculare.

\section{A}

WT En2-/-

B

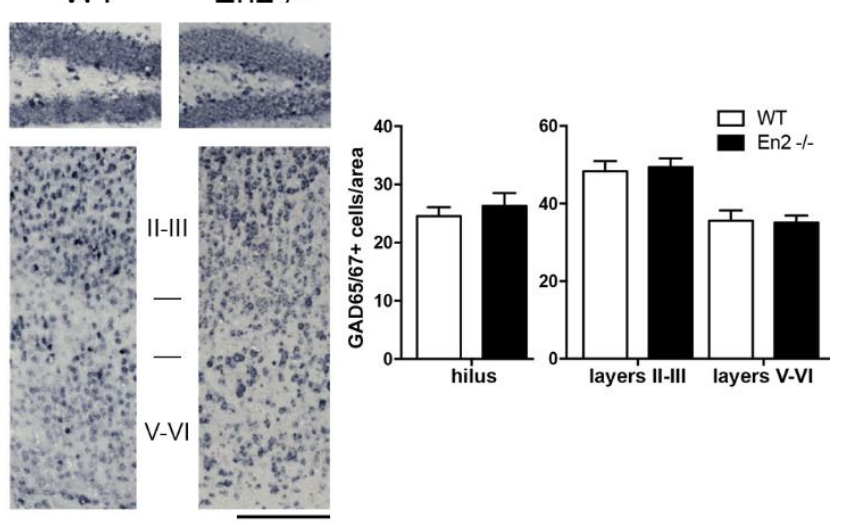

Figure 2. Unaltered number of GABAergic interneurons in the young En2 $2^{--}$hippocampus and somatosensory cortex. (A) Representative photomicrographs of in situ hybridizations showing GAD65/67 mRNA-positive neurons in the hilus (top) and somatosensory cortex (bottom) of P30 WT and $\mathrm{En2}^{--}$mice. Scale bar: $600 \mu \mathrm{m}$. Cortical layers are indicated in Roman numbers. (B) GABAergic (GAD65/67 mRNA-positive) interneuron cell counts from in situ hybridization experiments. Values are plotted as the mean number $\left( \pm\right.$ s.e.m) of positive cells per area $\left(0.1 \mathrm{~mm}^{2}\right.$ for the hilus and $0.12 \mathrm{~mm}^{2}$ for the somatosensory cortex) from 4 mice per genotype. Genotypes are as indicated. Abbreviations: hippo: hippocampus; ss ctx: somatosensory cortex. 
A
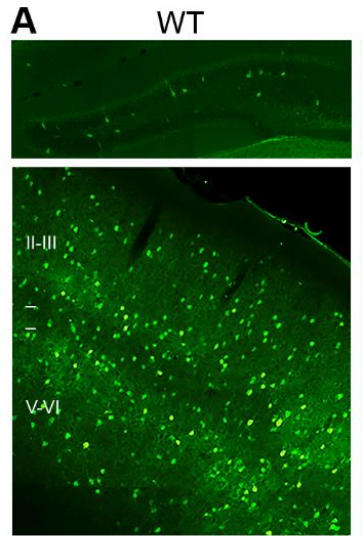

C

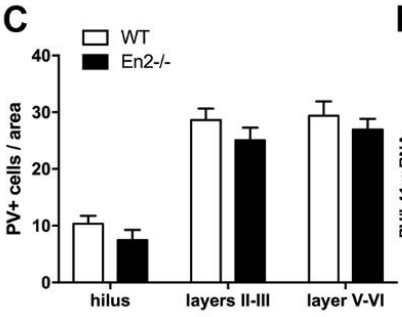

En2-I-
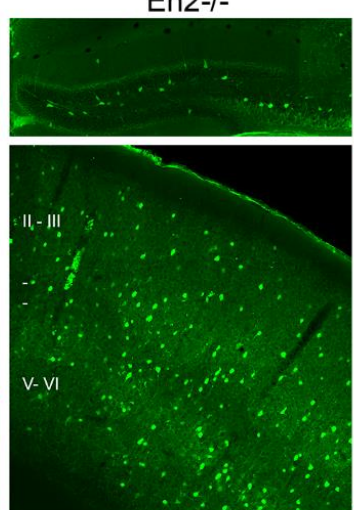

D
B
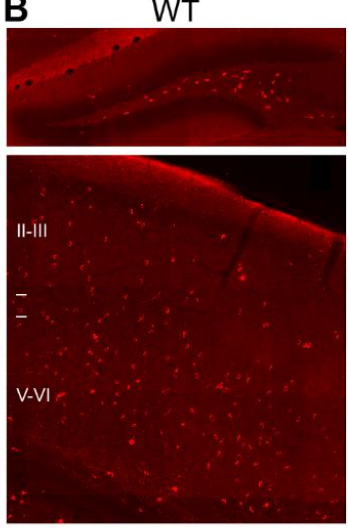

E
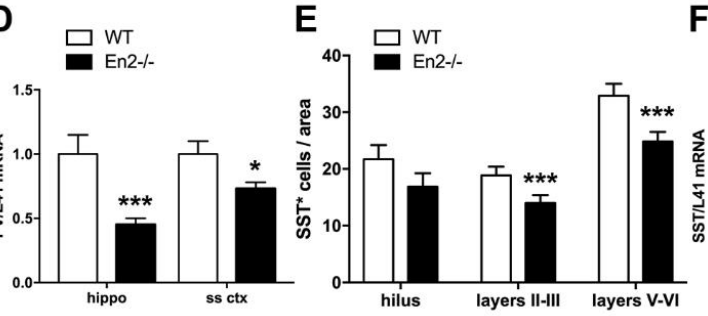

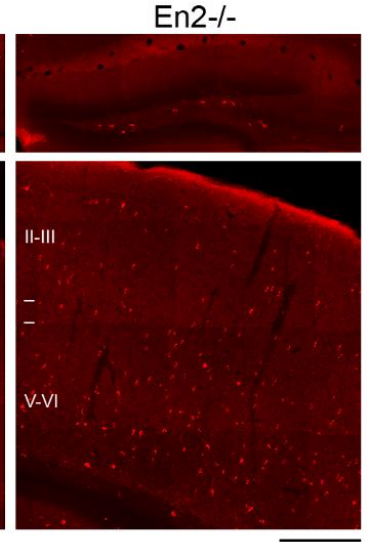

F 믈
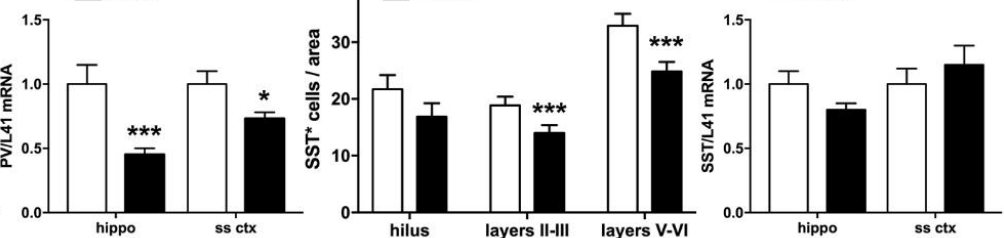

Figure 3. Reduced expression of parvalbumin (PV) and somatostatin (SST) interneuron markers in the young En2 $2^{--}$hippocampus and somatosensory cortex. (A,B) Representative immunostainings of PV ((A), green) and SST ((B), red) interneurons in the hilus (top) and somatosensory cortex (bottom) of P30 WT and En2 ${ }^{--}$mice. Cortical layers are indicated in Roman numbers. Scale bar: $400 \mu \mathrm{m}$. (C) PV cell counts from immunostaining experiments, plotted as the mean number ( \pm s.e.m) of positive cells per area $\left(0.1 \mathrm{~mm}^{2}\right.$ for the hilus and $0.12 \mathrm{~mm}^{2}$ for the somatosensory cortex) on 5 mice per genotype. (D) PV mRNA expression in the hippocampus and somatosensory cortex of P30 WT and En2 ${ }^{--}$mice. Values are plotted as the mean \pm s.e.m of PV/L41 comparative quantification ratio $\left({ }^{*} p<0.05\right.$; ${ }^{* * *} p<0.001$; Student's t-test, WT vs. En2 ${ }^{--}, 4 \mathrm{WT}$ and $6 \mathrm{En} 2^{--}$mice). (E) SST cell counts from immunostaining experiments, plotted as in $C{ }^{* * *} p<0.001$, two-way ANOVA followed by Sidak's multiple comparisons test, WT vs. En2 ${ }^{-}, 5$ mice per genotype). (F) SST mRNA expression in the hippocampus and somatosensory cortex of P30 WT and En2 ${ }^{--}$mice ( $\mathrm{n}=4 \mathrm{WT}$ and $6 \mathrm{En}^{-{ }^{-}}$). Values are plotted as in (D). Genotypes are as indicated. Abbreviations: PV, parvalbumin; SST, somatostatin.

Table 2 summarizes in situ hybridization, immunohistochemistry, and RT-PCR data on interneuron marker expression in the hippocampus and somatosensory cortex of P30 WT and En2 ${ }^{-}$mice. Taken together, these data indicate that, as already observed in adult animals, the En2 null mutation results in the altered expression of PV and SST interneuron markers in the hippocampus and somatosensory cortex of young mice.

Table 2. Expression of interneuron markers in the hippocampus and somatosensory cortex of En2 ${ }^{-/}$ P30 mice.

\begin{tabular}{cccccc}
\hline Interneuron Marker & \multicolumn{2}{c}{ Hippocampus } & \multicolumn{3}{c}{ Somatosensory Cortex } \\
\hline & mRNA & $\begin{array}{c}\text { Cell Counts } \\
\text { (hilus) }\end{array}$ & mRNA & $\begin{array}{c}\text { Cell Counts } \\
\text { (layers II-III) }\end{array}$ & $\begin{array}{c}\text { Cell Counts } \\
\text { (layers V-VI) }\end{array}$ \\
\hline GAD & not tested & no difference & not tested & no difference & no difference \\
PV & $-55 \%$ & no difference & $-27 \%$ & no difference & no difference \\
SST & no difference & no difference & no difference & $-25 \%$ & $-25 \%$ \\
\hline
\end{tabular}

Data are presented as the mean percentage of the reduction of interneuron marker mRNA and positive cells detected in En ${ }^{-/}$mice, as compared to WT littermates. See text and figures for experimental details. Abbreviations as in the text. 


\subsection{Altered Expression of GABA Receptor Subunits in Young and Adult En2 Mutant Mice}

Several human and animal studies indicate that altered GABA receptor subunit expression contributes to impaired GABAergic neurotransmission in $\operatorname{ASD}[10,25,26]$. In order to identify the potential mechanisms of GABA receptor dysfunction in our mouse model of ASD, we investigated the expression of GABA receptor subunits in the hippocampus and somatosensory cortex of P30 and adult WT and $E n 2^{--}$mice by using quantitative RT-PCR. Specifically, we analyzed the mRNA of several different subunits that mainly contribute to ionotropic $\mathrm{GABA}_{A}$ and metabotropic $\mathrm{GABA}_{B}$ receptors [27-29]: GABA $_{A}$ receptor subunits $\alpha 1, \alpha 2, \alpha 3, \alpha 5$ (Gabra1-5), $\beta 2, \beta 3$ (Gabrb2-3), $\gamma 2$ (Gabrg2), and $\mathrm{GABA}_{\mathrm{B}}$ receptor subunit $2(\mathrm{Gabbr})$.

At P30, Gabra5, Gabrab3, and Gabrg2 mRNAs were upregulated in the En2-- hippocampus, as compared to age-matched controls $\left(+30 \%,+30 \%\right.$, and $+43 \%$, respectively; ${ }^{* *} p<0.01$, Student's t-test, Figure $4 \mathrm{~A})$, while all other subunit mRNAs were unchanged ( $p>0.05$, Student's $t$-test, Figure $4 \mathrm{~A})$. In the somatosensory cortex, only Gabrab3 and Gabbr2 mRNAs significantly differed between the two genotypes (respectively, $+30 \%$ and $-26 \%$ in $E n 2^{--}$as compared with WT mice; ${ }^{* *} p<0.01$, Student's $t$-test, Figure 4A). The expression profile of GABA receptor subunits markedly differed during adulthood. In the somatosensory cortex, only Gabra1 mRNA was significantly lower in En2 $2^{--}$as compared with WT mice $\left(-17 \%\right.$; ${ }^{* *} p<0.01$, Student's $t$-test, Figure $\left.4 \mathrm{~B}\right)$, while all other subunit mRNAs were comparably expressed in both genotypes ( $p>0.05$, Student's $t$-test, Figure 4B). Finally, in the hippocampus, Gabra3, Gabrb2, Gabrb3, and Gabbr2 mRNAs significantly differed between the two genotypes (respectively, $+10 \%,-33 \%,-20 \%$, and $-10 \%$ in En2 ${ }^{--}$as compared with WT mice; ${ }^{*} p<0.05,{ }^{* *} p<0.01$, Student's $t$-test, Figure 4B), while the other subunit mRNAs remained unchanged ( $p>0.05$, Student's $t$-test, Figure 4B).
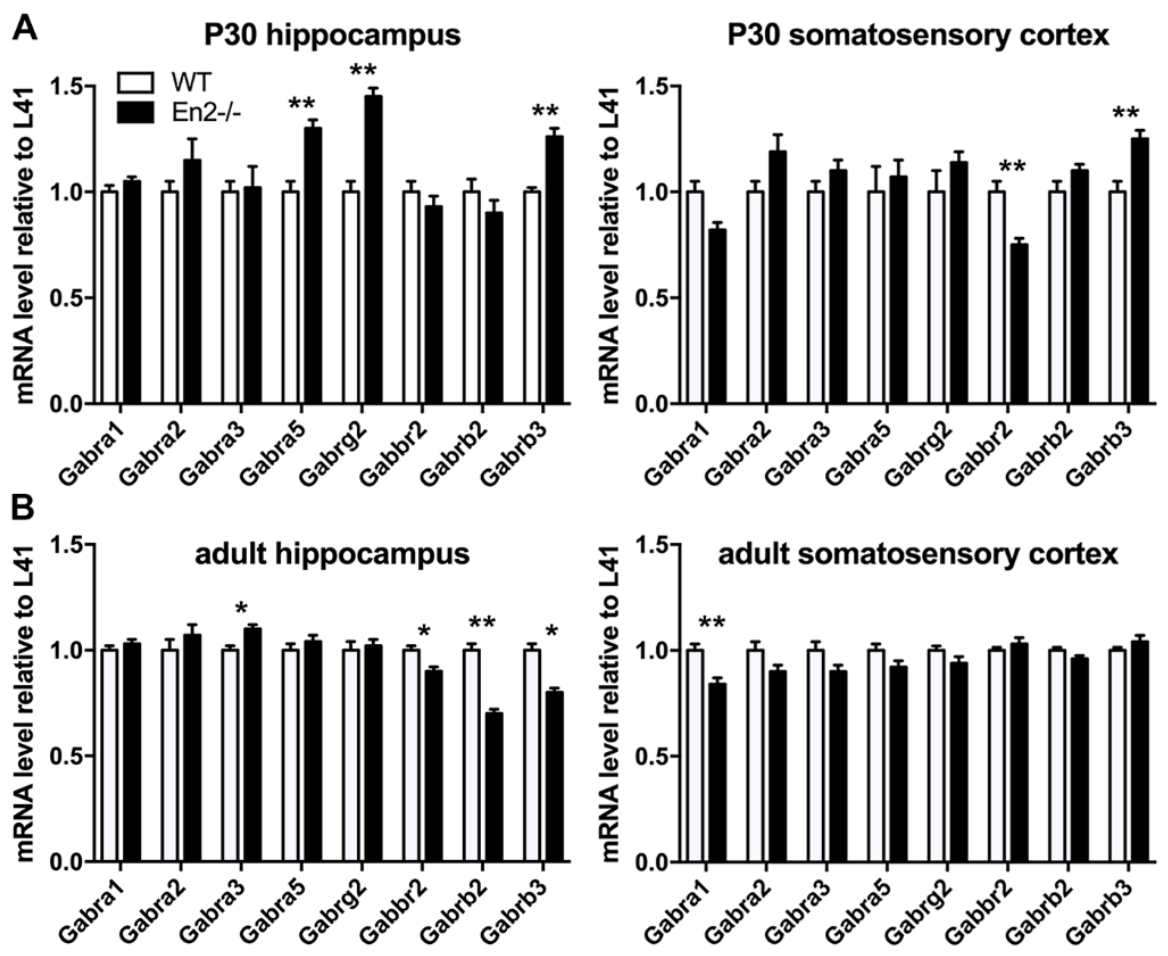

Figure 4. mRNA expression of GABA receptor subunits in the forebrain of young and adult WT and $E n 2^{-/-}$mice. (A,B) Relative mRNA expression level of GABA receptor subunits, as obtained by quantitative RT-PCR performed on hippocampus and somatosensory cortex of P30 (A) and adult (B) WT and $\mathrm{En}^{-{ }^{--}}$mice. Values are expressed as the mean \pm s.e.m of each subunit/L41 comparative quantification ratios (4 mice per age and genotype; ${ }^{*} p<0.05,{ }^{* *} p<0.01$, Student's $t$-test, WT vs. En2 ${ }^{-\sigma^{-}}$). Brain areas, ages, and genotypes are as indicated. Abbreviations are as in the text. 
Table 3 summarizes the expression profile of GABA receptor subunit mRNAs in the hippocampus and somatosensory cortex of P30 and adult WT and En2 ${ }^{--}$mice. Taken together, these data show that mice lacking En2 display an altered expression of several $\mathrm{GABA}_{\mathrm{A}}$ and $\mathrm{GABA}_{\mathrm{B}}$ receptor subunits in the hippocampus and somatosensory cortex of young and adult mice.

Table 3. mRNA expression of GABA receptor subunits in the hippocampus and somatosensory cortex of $E n 2^{-/-}$P30 and adult mice.

\begin{tabular}{ccccc}
\hline Receptor Subunit & \multicolumn{2}{c}{ P30 } & \multicolumn{2}{c}{ Adult } \\
\hline & Hippocampus & $\begin{array}{c}\text { Somatosensory } \\
\text { Cortex }\end{array}$ & Hippocampus & Somatosensory Cortex) \\
\hline Gabra1 & no difference & no difference & no difference & $-17 \%$ \\
Gabra2 & no difference & no difference & no difference & no difference \\
Gabra3 & no difference & no difference & $+10 \%$ & no difference \\
Gabra5 & $+30 \%$ & no difference & no difference & no difference \\
Gabrb2 & no difference & no difference & $-33 \%$ & no difference \\
Gabrb3 & $+30 \%$ & $+30 \%$ & $-20 \%$ & no difference \\
Gabrg2 & $+43 \%$ & no difference & no difference & no difference \\
Gabbr2 & no difference & $-26 \%$ & $-10 \%$ & no difference \\
\hline
\end{tabular}

Data are presented as the mean percentage of the reduction of GABA receptor subunit mRNAs in En2 ${ }^{--}$mice, as compared with WT littermates. See text and figures for experimental details. Abbreviations as in the text.

We previously showed that the protein levels of the $\mathrm{GABA}_{\mathrm{A}}$ receptor subunit $\beta 3$ were decreased in the adult $E n 2^{--}$hippocampus, thus confirming mRNA expression data [30]. Here, we investigated the protein expression of $\mathrm{GABA}_{\mathrm{A}}$ receptor subunits $\alpha 1, \alpha 3$, and $\alpha 5$ in the hippocampus of P30 WT and $E n 2^{--}$mice. Immunohistochemistry experiments showed a slight increase of GABRA1 and GABRA5 staining in the En2-- dentate gyrus, as compared to WT controls; conversely, GABRA3 staining was comparable in both genotypes (Figure 5).

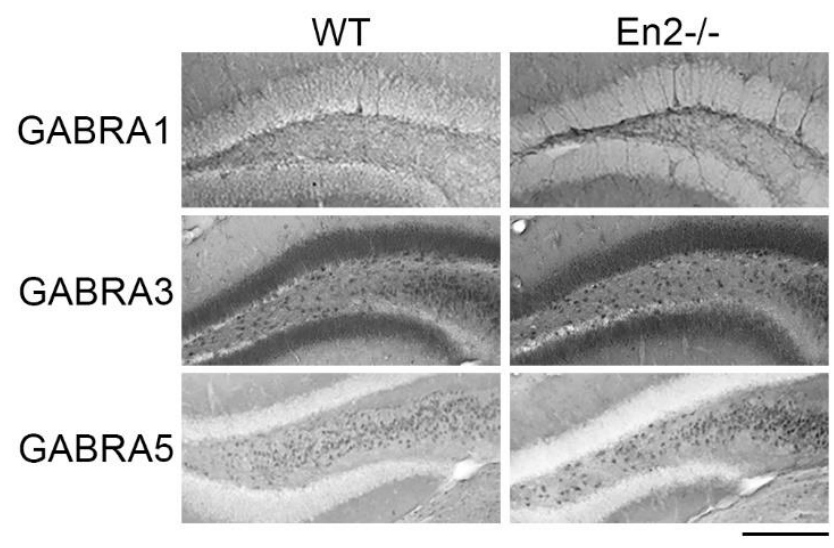

Figure 5. Expression of $\mathrm{GABA}_{\mathrm{A}}$ receptor subunits in dentate gyrus of young WT and $E n 2^{--}$mice. Pictures show representative immunostainings of GABRA1, GABRA3, and GABRA5 subunits in the dentate gyrus of P30 WT and En2 ${ }^{--}$mice. Scale bar: $300 \mu \mathrm{m}$. Genotypes are as indicated. Abbreviations are as in the text.

\section{Discussion}

\subsection{Brief Summary of Results}

In this study, we showed that young (P30) En2 ${ }^{-/-}$mice display an altered expression of interneuron markers, namely, a reduced expression in PV mRNA in the hippocampus and somatosensory cortex and a reduced number of SST-positive cells in the somatosensory cortex. The expression of GABA receptor subunit mRNAs was also altered in these brain areas of young and adult En2 mutants. The GABA receptor $\beta 3$ subunit (Gabrb3) was the most severely affected, showing a significant upregulation in both 
areas at P30, and a downregulation in the adult hippocampus. Among the other subunits, $\alpha 5$ showed a consistent increase of both mRNA and protein levels in the hippocampus of young En2 mutant mice.

\subsection{Altered Expression of GABAergic Interneuron Markers in Young En2 Mutant Mice}

Previous studies from our laboratory showed a reduced expression of GABAergic interneuron markers in the forebrain of adult En $2^{-/}$mice. The present results, obtained from P30 mice, expand this notion and confirm that En2 contributes to regulate the function of forebrain GABAergic interneurons already at early postnatal ages. As previously observed in adult mice [22], the loss of En2 resulted in the reduced expression of PV and SST in the hippocampus and somatosensory cortex of young P30 mice, without affecting the total number of GAD-positive interneurons, nor the layering of these brain areas. More specifically, a 25\% reduction in SST-positive cells was consistently detected in layers II-III of P30 (this study) and adult [22] En2 ${ }^{--}$mice. This data confirms that the En2 mutation impacts the expression of specific GABAergic interneuron markers during postnatal development. This selective effect is likely due to altered embryonic development of GABAergic interneurons in En2 mutant brains. Indeed, neural stem cells derived from embryonic En $2^{-/}$basal forebrain are not capable to properly differentiate into mature neurons, and do show a reduced expression of PV and SST GABAergic markers during in vitro differentiation [31]. However, the expression profile of PV and SST in En2 mutant brains significantly differed between P30 and adult mice. Although both PV mRNA and cell number were shown to be reduced in the adult En2-- hippocampus and somatosensory cortex [22], only PV mRNA (but not cell number) was decreased in the same brain areas of young En2 mutants. Similarly, SST mRNA downregulation was detected only in the adult [22] but not P30 hippocampus. Interestingly, the downregulation of PV expression in the absence of a PV cell loss has been observed in other mouse models of ASD such as Cntnap $2^{--}$and Shank3b $b^{--}$mice, as well as mice exposed in utero to valproic acid. In all these models, no loss of PV neurons was detected in ASD-relevant brain regions including the striatum, whereas PV protein levels were decreased in this area [32-34]. The authors of these studies proposed that the observed downregulation of PV in the striatum of these mouse models might represent a homeostatic mechanism to counteract reduced inhibition, thus to maintain a physiological excitation/inhibition balance [34,35]. A similar mechanism might therefore be in place in the hippocampus and somatosensory cortex of young En2 mutants, which showed reduced PV mRNA (but not cell number) in these same brain areas. Taken together, these results strengthen the notion that specific markers of hippocampal and cortical interneurons are affected in mouse models of ASD during postnatal development.

\subsection{Altered Expression of GABA Receptor Subunits in Young and Adult En2 Mutant Mice}

Abnormal GABAergic inhibition has been proposed as a pathogenic mechanism of multiple neurodevelopmental disorders, including ASD [10]. As mentioned above, GABAergic interneurons are clearly affected in human ASD, as shown by post-mortem studies performed on hippocampal tissues from ASD patients compared to control samples from typically-developing subjects [11]. Similar results have been obtained in mouse models of ASD [10]. GABA receptor dysfunction has also been detected in ASD subjects and mouse models. For example, reduced ionotropic $\mathrm{GABA}_{\mathrm{A}}$ receptor subunits and benzodiazepine binding were detected in the cerebral cortex of ASD individuals [36-40]. Among the several different $\mathrm{GABA}_{\mathrm{A}}$ receptors, the $\alpha 5$ subtype gained the attention of ASD researchers. Single nucleotide polymorphisms (SNPs) across the GABRA5 and GABRB3 genes (respectively, coding for the $\alpha 5$ and $\beta 3$ subunits of the $\mathrm{GABA}_{\mathrm{A}} \alpha 5$ receptor) are associated with ASD [41,42]. A reduced density of $\mathrm{GABA}_{\mathrm{A}} \alpha 5$ receptors was initially detected in the brain of ASD patients [43], a result that was not replicated in further studies [44]. Mice lacking the $\beta 3$ subunit of the $\mathrm{GABA}_{\mathrm{A}}$ receptor (Gabrb3 ${ }^{--}$mice) show ASD-relevant traits such as reduced sociability [45] and abnormal response to tactile stimulation [46], thus strengthening the importance of the $\mathrm{GABA}_{\mathrm{A}} \alpha 5$ receptor subtype in ASD pathogenesis. 
In the present study, we analyzed the expression of $\mathrm{GABA}_{\mathrm{A}}$ and $\mathrm{GABA}_{\mathrm{B}}$ receptor subunit mRNAs in the hippocampus and somatosensory cortex of P30 and adult En2 ${ }^{-}$and WT mice. With the exception of Gabra2, all other subunit mRNAs were deregulated in at least one structure and at one age of the two analyzed (Table 3). Interestingly, the most consistently deregulated was Gabrb3 mRNA, which codes for the $\beta 3$ subunit of the $\mathrm{GABA}_{\mathrm{A}}$ receptor. Gabrb3 mRNA was upregulated in the En $2^{-/}$hippocampus and somatosensory cortex at P30, while in adult En2 ${ }^{--}$mice, it showed a significant downregulation only in the hippocampus. These results indicate that Gabrb3 mRNA in the hippocampus undergoes a time-dependent regulation in En2 ${ }^{-/}$mice; in addition, the Gabrb3 mRNA upregulation has never been observed in other mouse models of ASD. More importantly, the results obtained in adult mice are in agreement with our previous study that showed a marked downregulation of the FMRP and GABRB3 protein in the adult En2 ${ }^{-/}$hippocampus [30]. In accordance with these results, reduced levels of FMRP and GABRB3 proteins were detected in the cerebellum of adult ASD subjects [38]. Interestingly, mice lacking FMRP (Fmr1 ${ }^{-/}$mice), which share anatomical and functional abnormalities with En2 mutants [19,47,48], also show a decreased expression of GABA receptor subunit mRNAs and proteins $[49,50]$. Thus, FMRP-dependent downregulation of $\mathrm{GABA}_{\mathrm{A}} \beta 3$ subunit expression might contribute to alter GABAergic transmission in En2 mutant mice. Conversely, the $\alpha 5$ subunit showed a consistent increase in both mRNA and protein levels in the hippocampus of young En2 mutant mice. Increased levels of Gabra5 mRNA have been reported in the cerebellum of ASD subjects, whereas a reduced expression was detected in neocortical areas [37]. In keeping with these findings, $\mathrm{GABA}_{\mathrm{A}}$ receptors containing the $\alpha 5$ subunit were initially proposed as a potential pharmacological target in ASD [43], although these results were not corroborated by subsequent studies [44]. Regarding the expression of the $\mathrm{GABA}_{\mathrm{A}} \alpha 1$ subunit, we reported discordant results between its mRNA and protein levels in the hippocampus of P30 En2 mutants (Figures 4 and 5). Different mechanisms, including the downregulation of microRNAs inhibiting GABRA1 protein translation, or post-translational modifications altering its trafficking, might be involved. Interestingly, studies performed on post-mortem brain samples from ASD subjects also reported discordant mRNA and protein expression profiles of GABA receptor subunits [37].

Finally, in agreement with previous studies on ASD mouse models [15,50] and human subjects [37], GABA receptor subunits expression varied across different brain regions in En2 mutants, and the detected changes between WT and $\mathrm{En}^{2-}$ mice were relatively small. Nevertheless, the entity of the expression changes detected in En2 mutants (ranging from 10\% to 43\%) was comparable to what observed in Fmr1 knockout mice [50], which definitely show compromised GABAergic transmission [51]. We therefore speculate that these alterations might be enough to modify GABA signaling, thus substantiating a pathological readout also in $E n 2^{-/}$mice.

\section{Conclusions}

Previous studies from our laboratory showed that the expression of several markers of GABAergic synaptic function (receptors, structural proteins, signaling molecules, etc.) are deregulated in the forebrain of En2 $2^{--}$mice compared to WT littermates $[18,22,23]$. The results reported in the present study confirm these observations, strengthening the notion that GABAergic dysfunction is a common feature of several mouse models of ASD. Most importantly, our results indicate that GABAergic signaling dysfunction might contribute the complex behavioral phenotype of En2 mutants since early postnatal ages. Together with other studies addressing early postnatal deficits of GABA transmission in ASD mouse models [49,52-54], our findings might guide future research towards the identification of precocious markers of GABAergic dysfunction in ASD.

Author Contributions: Conceptualization, G.P., P.S., S.C., and Y.B.; Investigation, G.P., A.G., M.M., M.P., and P.S.; Data Curation, G.P., A.G., M.M., M.P., P.S., and Y.B.; Writing-Original Draft Preparation, Y.B.; Writing-Review \& Editing, P.S., S.C., G.P., and Y.B.; Supervision, G.P., P.S., and Y.B.; Funding Acquisition, P.S., S.C., and Y.B. All authors have read and agreed to the published version of the manuscript. 
Funding: This research was funded by the Italian Ministry of University and Research (PRIN 2008 grant \# 200894SYW2_002 and PRIN 2010-2011 grant \# 2010N8PBAA_002 to Y.B.), University of Trento start-up grant (S.C.), and University of Trento strategic project TRAIN-Trentino Autism Initiative (https://projects.unitn.it/train/ index.html) to Y.B. G.P. was supported by a post-doctoral fellowship of Fondazione Veronesi (Milan, Italy). P.S. was supported by Provincia Autonoma di Trento (Italy) under the Marie Curie-People COFUND action of the European Community.

Acknowledgments: We thank the administrative staff of CIMeC, Department CIBIO and CNR Neuroscience Institute for support.

Conflicts of Interest: The authors declare no conflicts of interest.

\section{References}

1. Rubenstein, J.L.R.; Merzenich, M.M. Model of autism: Increased ratio of excitation/inhibition in key neural systems. Genes Brain Behav. 2003, 2, 255-267. [CrossRef] [PubMed]

2. Wonders, C.P.; Anderson, S.A. The origin and specification of cortical interneurons. Nat. Rev. Neurosci. 2006, 7, 687-696. [CrossRef] [PubMed]

3. Ascoli, G.A.; Alonso-Nanclares, L.; Anderson, S.A.; Barrionuevo, G.; Benavides-Piccione, R.; Burkhalter, A.; Buzsáki, G.; Cauli, B.; Defelipe, J.; Fairén, A.; et al. Petilla terminology: Nomenclature of features of GABAergic interneurons of the cerebral cortex. Nat. Rev. Neurosci. 2008, 9, 557-568.

4. Kepecs, A.; Fishell, G. Interneuron cell types are fit to function. Nature 2014, 505, 318-326. [CrossRef]

5. Tremblay, R.; Lee, S.; Rudy, B. GABAergic interneurons in the neocortex: From cellular properties to circuits. Neuron 2016, 91, 260-292. [CrossRef]

6. Pelkey, K.A.; Chittajallu, R.; Craig, M.T.; Tricoire, L.; Wester, J.C.; McBain, C.J. Hippocampal GABAergic inhibitory interneurons. Physiol. Rev. 2017, 97, 1619-1747. [CrossRef]

7. Huang, Z.J.; Paul, A. The diversity of GABAergic neurons and neural communication elements. Nat. Rev. Neurosci. 2019, 20, 563-572. [CrossRef]

8. Rossignol, E. Genetics and function of neocortical GABAergic interneurons in neurodevelopmental disorders. Neural. Plast. 2011, 2011, 649325. [CrossRef]

9. Marín, O. Interneuron dysfunction in psychiatric disorders. Nat. Rev. Neurosci. 2012, 13, 107-120. [CrossRef]

10. Bozzi, Y.; Provenzano, G.; Casarosa, S. Neurobiological bases of autism-epilepsy comorbidity: A focus on excitation/inhibition imbalance. Eur. J. Neurosci. 2018, 47, 534-548. [CrossRef]

11. Lawrence, Y.A.; Kemper, T.L.; Bauman, M.L.; Blatt, G.J. Parvalbumin-, calbindin-, and calretinin-immunoreactive hippocampal interneuron density in autism. Acta Neurol. Scand. 2010, 121, $99-108$. [CrossRef]

12. Blatt, G.J.; Fatemi, S.H. Alterations in GABAergic biomarkers in the autism brain: Research findings and clinical implications. Anat. Rec. 2011, 294, 1646-1652. [CrossRef]

13. Benayed, R.; Choi, J.; Matteson, P.G.; Gharani, N.; Kamdar, S.; Brzustowicz, L.M.; Millonig, J.H. Autism-associated haplotype affects the regulation of the homeobox gene, ENGRAILED 2. Biol. Psychiatry 2009, 66, 911-917. [CrossRef]

14. Joyner, A.L.; Herrup, K.; Auerbach, B.A.; Davis, C.A.; Rossant, J. Subtle cerebellar phenotype in mice homozygous for a targeted deletion of the En-2 homeobox. Science 1991, 251, 1239-1243. [CrossRef]

15. DiCicco-Bloom, E.; Lord, C.; Zwaigenbaum, L.; Courchesne, E.; Dager, S.R.; Schmitz, C.; Schultz, R.T.; Crawley, J.; Young, L.J. The developmental neurobiology of autism spectrum disorder. J. Neurosci. 2006, 26, 6897-6906. [CrossRef]

16. Cheh, M.A.; Millonig, J.H.; Roselli, L.M.; Ming, X.; Jacobsen, E.; Kamdar, S.; Wagner, G.C. En2 knockout mice display neurobehavioral and neurochemical alterations relevant to autism spectrum disorder. Brain Res. 2006, 1116, 166-176. [CrossRef]

17. Brielmaier, J.; Matteson, P.G.; Silverman, J.L.; Senerth, J.M.; Kelly, S.; Genestine, M.; Millonig, J.H.; Dicicco-Bloom, E.; Crawley, J.N. Autism-relevant social abnormalities and cognitive deficits in engrailed-2 knockout mice. PLoS ONE 2012, 7, e40914. [CrossRef]

18. Provenzano, G.; Pangrazzi, L.; Poli, A.; Pernigo, M.; Sgadò, P.; Genovesi, S.; Zunino, G.; Berardi, N.; Casarosa, S.; Bozzi, Y. Hippocampal dysregulation of neurofibromin-dependent pathways is associated with impaired spatial learning in engrailed 2 knock-out mice. J. Neurosci. 2014, 34, 13281-13288. [CrossRef] 
19. Chelini, G.; Zerbi, V.; Cimino, L.; Grigoli, A.; Markicevic, M.; Libera, F.; Robbiati, S.; Gadler, M.; Bronzoni, S.; Miorelli, S.; et al. Aberrant somatosensory processing and connectivity in mice lacking Engrailed-2. J. Neurosci. 2019, 39, 1525-1538. [CrossRef]

20. Tripathi, P.P.; Sgadò, P.; Scali, M.; Viaggi, C.; Casarosa, S.; Simon, H.H.; Vaglini, F.; Corsini, G.U.; Bozzi, Y. Increased susceptibility to kainic acid-induced seizures in Engrailed-2 knockout mice. Neuroscience 2009, 159, 842-849. [CrossRef]

21. Sgadò, P.; Provenzano, G.; Dassi, E.; Adami, V.; Zunino, G.; Genovesi, S.; Casarosa, S.; Bozzi, Y. Transcriptome profiling in engrailed-2 mutant mice reveals common molecular pathways associated with autism spectrum disorders. Mol. Autism. 2013, 4, 51. [CrossRef]

22. Sgadò, P.; Genovesi, S.; Kalinovsky, A.; Zunino, G.; Macchi, F.; Allegra, M.; Murenu, E.; Provenzano, G.; Tripathi, P.P.; Casarosa, S.; et al. Loss of GABAergic neurons in the hippocampus and cerebral cortex of Engrailed-2 null mutant mice: Implications for autism spectrum disorders. Exp. Neurol. 2013, 247, 496-505. [CrossRef]

23. Allegra, M.; Genovesi, S.; Maggia, M.; Cenni, M.C.; Zunino, G.; Sgadò, P.; Caleo, M.; Bozzi, Y. Altered GABAergic markers, increased binocularity and reduced plasticity in the visual cortex of Engrailed-2 knockout mice. Front. Cell. Neurosci. 2014, 8, 163. [CrossRef]

24. Provenzano, G.; Corradi, Z.; Monsorno, K.; Fedrizzi, T.; Ricceri, L.; Scattoni, M.L.; Bozzi, Y. Comparative gene expression analysis of two mouse models of autism: Transcriptome profiling of the BTBR and En2 (-/-) Hippocampus. Front. Neurosci. 2016, 10, 396. [CrossRef]

25. Cellot, G.; Cherubini, E. GABAergic signaling as therapeutic target for autism spectrum disorders. Front Pediatr 2014, 2, 70. [CrossRef]

26. Braat, S.; Kooy, R.F. The GABAA Receptor as a therapeutic target for neurodevelopmental disorders. Neuron 2015, 86, 1119-1130. [CrossRef]

27. Fritschy, J.M.; Brünig, I. Formation and plasticity of GABAergic synapses: Physiological mechanisms and pathophysiological implications. Pharmacol. Ther. 2003, 98, 299-323. [CrossRef]

28. Olsen, R.W.; Sieghart, W. International Union of Pharmacology, LXX. Subtypes of gamma-aminobutyric acid(A) receptors: Classification on the basis of subunit composition, pharmacology, and function. Update. Pharmacol. Rev. 2008, 60, 243-260. [CrossRef]

29. Gassmann, M.; Bettler, B. Regulation of neuronal GABA(B) receptor functions by subunit composition. Nat. Rev. Neurosci. 2012, 13, 380-394. [CrossRef]

30. Provenzano, G.; Sgadò, P.; Genovesi, S.; Zunino, G.; Casarosa, S.; Bozzi, Y. Hippocampal dysregulation of FMRP/mGluR5 signaling in engrailed-2 knockout mice: A model of autism spectrum disorders. Neuroreport 2015, 26, 1101-1105. [CrossRef]

31. Boschian, C.; Messina, A.; Bozza, A.; Castellini, M.E.; Provenzano, G.; Bozzi, Y.; Casarosa, S. Impaired neuronal differentiation of neural stem cells lacking the Engrailed-2 gene. Neuroscience 2018, 386, 137-149. [CrossRef] [PubMed]

32. Filice, F.; Vörckel, K.J.; Sungur, A.Ö.; Wöhr, M.; Schwaller, B. Reduction in parvalbumin expression not loss of the parvalbumin-expressing GABA interneuron subpopulation in genetic parvalbumin and shank mouse models of autism. Mol. Brain 2016, 9, 10. [CrossRef]

33. Lauber, E.; Filice, F.; Schwaller, B. Prenatal valproate exposure differentially affects parvalbumin-expressing neurons and related circuits in the cortex and striatum of mice. Front. Mol. Neurosci. 2016, 9, 150. [CrossRef]

34. Lauber, E.; Filice, F.; Schwaller, B. Dysregulation of parvalbumin expression in the cntnap2-/- mouse model of autism spectrum disorder. Front. Mol. Neurosci. 2018, 11, 262. [CrossRef]

35. Lauber, E.; Filice, F.; Schwaller, B. Parvalbumin neurons as a hub in autism spectrum disorders. J. Neurosci. Res. 2018, 96, 360-361. [CrossRef]

36. Fatemi, S.H.; Reutiman, T.J.; Folsom, T.D.; Thuras, P.D. GABA(A) receptor downregulation in brains of subjects with autism. J. Autism. Dev. Disord. 2009, 39, 223-230. [CrossRef]

37. Fatemi, S.H.; Reutiman, T.J.; Folsom, T.D.; Rooney, R.J.; Patel, D.H.; Thuras, P.D. mRNA and protein levels for GABAA alpha4, alpha5, beta1 and GABABR1 receptors are altered in brains from subjects with autism. J. Autism. Dev. Disord. 2010, 40, 743-750. [CrossRef]

38. Fatemi, S.H.; Folsom, T.D.; Kneeland, R.E.; Liesch, S.B. Metabotropic glutamate receptor 5 upregulation in children with autism is associated with underexpression of both Fragile $\mathrm{X}$ mental retardation protein and GABAA receptor beta 3 in adults with autism. Anat. Rec. 2011, 294, 1635-1645. [CrossRef] 
39. Oblak, A.; Gibbs, T.T.; Blatt, G.J. Decreased GABAA receptors and benzodiazepine binding sites in the anterior cingulate cortex in autism. Autism. Res. 2009, 2, 205-219. [CrossRef]

40. Oblak, A.; Gibbs, T.T.; Blatt, G.J. Reduced GABAA receptors and benzodiazepine binding sites in the posterior cingulate cortex and fusiform gyrus in autism. Brain Res. 2011, 1380, 218-228. [CrossRef]

41. McCauley, J.L.; Olson, L.M.; Delahanty, R.; Amin, T.; Nurmi, E.L.; Organ, E.L.; Jacobs, M.M.; Folstein, S.E.; Haines, J.L.; Sutcliffe, J.S. A linkage disequilibrium map of the 1-Mb 15q12 GABA(A) receptor subunit cluster and association to autism. Am. J. Med. Genet. B Neuropsychiatr. Genet. 2004, 131B, 51-59. [CrossRef] [PubMed]

42. Delahanty, R.J.; Kang, J.Q.; Brune, C.W.; Kistner, E.O.; Courchesne, E.; Cox, N.J.; Cook, E.H., Jr.; Macdonald, R.L.; Sutcliffe, J.S. Maternal transmission of a rare GABRB3 signal peptide variant is associated with autism. Mol. Psychiatry 2011, 16, 86-96. [CrossRef] [PubMed]

43. Mendez, M.A.; Horder, J.; Myers, J.; Coghlan, S.; Stokes, P.; Erritzoe, D.; Howes, O.; Lingford-Hughes, A.; Murphy, D.; Nutt, D. The brain GABA-benzodiazepine receptor alpha-5 subtype in autism spectrum disorder: A pilot [(11)C]Ro15-4513 positron emission tomography study. Neuropharmacology 2013, 68, 195-201. [CrossRef]

44. Horder, J.; Andersson, M.; Mendez, M.A.; Singh, N.; Tangen, Ä.; Lundberg, J.; Gee, A.; Halldin, C.; Veronese, M.; Bölte, S.; et al. GABA(A) receptor availability is not altered in adults with autism spectrum disorder or in mouse models. Sci. Transl. Med. 2018, 10, eaam8434. [CrossRef]

45. DeLorey, T.M.; Sahbaie, P.; Hashemi, E.; Homanics, G.E.; Clark, J.D. Gabrb3 gene deficient mice exhibit impaired social and exploratory behaviors, deficits in non-selective attention and hypoplasia of cerebellar vermal lobules: A potential model of autism spectrum disorder. Behav. Brain Res. 2008, 187, 207-220. [CrossRef]

46. Orefice, L.L.; Zimmerman, A.L.; Chirila, A.M.; Sleboda, S.J.; Head, J.P.; Ginty, D.D. Peripheral mechanosensory neuron dysfunction underlies tactile and behavioral deficits in mouse models of ASDs. Cell 2016, 166, 299-313. [CrossRef]

47. Ellegood, J.; Anagnostou, E.; Babineau, B.A.; Crawley, J.N.; Lin, L.; Genestine, M.; DiCicco-Bloom, E.; Lai, J.K.; Foster, J.A.; Peñagarikano, O; et al. Clustering autism: Using neuroanatomical differences in 26 mouse models to gain insight into the heterogeneity. Mol. Psychiatry 2015, 20, 118-125. [CrossRef]

48. Zerbi, V.; Ielacqua, G.D.; Markicevic, M.; Haberl, M.G.; Ellisman, M.H.; A-Bhaskaran, A.; Frick, A.; Rudin, M.; Wenderoth, N. Dysfunctional autism risk genes cause circuit-specific connectivity deficits with distinct developmental trajectories. Cereb. Cortex 2018, 28, 2495-2506. [CrossRef]

49. Adusei, D.C.; Pacey, L.K.; Chen, D.; Hampson, D.R. Early developmental alterations in gabaergic protein expression in Fragile X knockout mice. Neuropharmacology 2010, 59, 167-171. [CrossRef]

50. D'Hulst, C.; De Geest, N.; Reeve, S.P.; Van Dam, D.; De Deyn, P.P.; Hassan, B.A.; Kooy, R.F. Decreased expression of the GABAA receptor in fragile X syndrome. Brain Res. 2006, 1121, 238-245. [CrossRef]

51. Chau, D.K.; Choi, A.Y.; Yang, W.; Leung, W.N.; Chan, C.W. Downregulation of glutamatergic and GABAergic proteins in valproic acid associated social impairment during adolescence in mice. Behav. Brain Res. 2017, 316, 255-260. [CrossRef]

52. Van der Aa, N.; Kooy, R.F. GABAergic abnormalities in the fragile X syndrome. Eur. J. Paediatr. Neurol. 2020, 24, 100-104. [CrossRef]

53. Leonzino, M.; Busnelli, M.; Antonucci, F.; Verderio, C.; Mazzanti, M.; Chini, B. The timing of the excitatory-to-inhibitory GABA switch is regulated by the oxytocin receptor via KCC2. Cell Rep. 2016, 15, 96-103. [CrossRef]

54. Modi, B.; Pimpinella, D.; Pazienti, A.; Zacchi, P.; Cherubini, E.; Griguoli, M. Possible implication of the CA2 hippocampal circuit in social cognition deficits observed in the neuroligin 3 knock-out mouse, a non-syndromic animal model of autism. Front. Psychiatry 2019, 10, 513. [CrossRef]

(C) 2020 by the authors. Licensee MDPI, Basel, Switzerland. This article is an open access article distributed under the terms and conditions of the Creative Commons Attribution (CC BY) license (http://creativecommons.org/licenses/by/4.0/). 